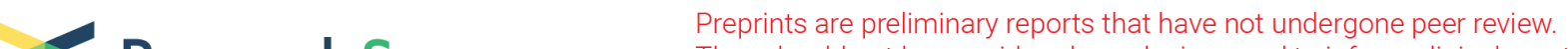 Research Square \\ Comparison with Tube Core and Magill forceps for Nasotracheal Intubation: A Randomized Controlled Trial
}

Rui Hu

Department of Anesthesiology,The Third Affiliated Hospital of Anhui Medical University Jingyi Niu

Department of Anesthesiology,The Third Affiliated Hospital of Anhui Medical University

Lining Wu

Department of Anesthesiology,The Third Hospital of Anhui Medical University

Hao Sun

Department of Anesthesiology,The Third Affiliated Hospital of Anhui Medical University

\section{Peng Sun}

Department of Anesthesiology,The Third Affiliated of Anhui Medical University

\section{Jiaying Huang}

Department of Otorhinolaryngology Surgery,The Third Affiliated Hospital of Anhui Medical University Junma Yu ( $\triangle$ majuny163@163.com )

The Third Affiliated Hospital of Anhui Medical University https://orcid.org/0000-0003-4053-5942

\section{Research article}

Keywords: Nasotracheal intubation, Magill forceps, Airway management

Posted Date: October 5th, 2020

DOl: https://doi.org/10.21203/rs.3.rs-72103/v1

License: (c) (i) This work is licensed under a Creative Commons Attribution 4.0 International License. Read Full License 


\section{Abstract}

Background: Magill forceps are frequently used to complete the process of nasotracheal intubation (NTI). We aimed to identify a tube core that could facilitate the NTI process conveniently without Magill forceps.

Methods: Sixty patients with no differences between the two groups (30 per group) with regard to demographic data were enrolled in our study. In Group $M$, the wire-reinforced tracheal tube was inserted into the trachea using Magill forceps. However, in Group T, a tube core bent to the physiological curve of the nasal cavity lubricated with aseptic liquid paraffin was inserted into the tracheal tube, and the tube core was then withdrawn after the tracheal tube was advanced through the glottis under direct vision.

Results: All NTIs were completed successfully, and no Magill forceps were used in Group T. There was a significant difference in total NTI time between the two groups (Group M, 59.7 (5.1) sec vs Group T, 52.4 (3.1) sec). Mild epistaxis was observed in 6 patients in Group M and 5 patients in Group T (6/30 vs 5/30, respectively). There was no presence of damaged oral tissue or damage to the teeth in either group.

Conclusion: We conclude that using the tube core, which is a disposable sterilised stylet, for NTI is a convenient choice.

Trial registrations: This clinical research was registered at the Chinese Clinical Trial Registry (www.Chictr.org.cn, ChiCTR1900027387).

\section{Background}

Nasotracheal intubation (NTI) is widely used in the clinic for oral and maxillofacial surgeries. Magill forceps have been frequently used to complete the process of NTI in several previous studies[1-4]. However, this instrument may cause rupture of the cuff or mucosal injury and even lead to infection[5-7].

Softer tracheal tubes such as reinforced ones tend to move along the posterior pharyngeal wall rather towards the laryngeal inlet, and it is difficult to navigate them into the vocal cord without using Magill forceps[8]. It was reported that a rigid wire tube body bent along the radian of the nasal cavity could help the anaesthesiologist complete NTI [9] more easily. In another study, the use of a stylet resulted in significantly higher first-attempt intubation success among patients undergoing endotracheal intubation compared with use of a bougie[10]. The tube core, a malleable rigid stylet, was similar to the wire tube body (Fig. 1, A). Thus, we hypothesised that a tube core could facilitate the NTI process conveniently without the use of Magill forceps.

\section{Methods}

This study protocol was approved by the Institutional Research Ethics Committee of The First People's Hospital of Hefei (No. 2019-12) and was registered in the Chinese Clinical Trial Registry 
(www.Chictr.org.cn, ChiCTR1900027387). Written informed consent was obtained from all patients, and the study was conducted in accordance with the Declaration of Helsinki.

We excluded patients from our study if they fell into any of the following categories: (1) age younger than 18 years or older than 80 years; (2) a body mass index (BMI) $\geq 30 \mathrm{~kg} / \mathrm{m}^{2}$; (3) the Cormack and Lehane (CL) grading scale of the laryngoscopic view of 3 or 4[11]; (4) a history of nasal abnormality (e.g., nasal trauma, surgery, obstruction, and polyps); (5) current anticoagulation therapy; (6) the presence of an oral malignant tumour or difficulty anticipated in airway management; (7) a mental disorder diagnosis; and (8) cervical vertebra instability, trauma or rheumatoid arthritis. None of the patients were premedicated, and standard monitoring equipment, such as electrocardiograms, noninvasive arterial blood pressure measurement devices and continuous pulse oximeters, was used in the operating room. All study subjects were randomised, using computer-generated random numbers, and envelopes containing randomisation numbers were used to allocate the patients to the following two groups $(n=30$ per group) according to the airway device that would be used to guide NTI: Magill forceps group (Group M) and tube core group (Group T).

An otorhinolaryngologist who was blinded to the group assignment used a nasal speculum to check for abnormalities inside the nostrils and to select the more patent nostril. If the patency of both nostrils was equal, NTI was performed in the right nostril[3]. General anaesthesia was induced with 1.5-2 mg/kg intravenous propofol and $0.3 \mu \mathrm{g} / \mathrm{kg}$ sufentanil, and muscle relaxation was achieved by intravenous administration of $0.15 \mathrm{mg} / \mathrm{kg}$ cisatracurium. Before intubation, manual ventilation was performed with $100 \%$ oxygen through a facemask for 3 min. The selected nostril was packed with gauze containing epinephrine to prevent bleeding. Males and females were intubated with 6.5-mm and 6.0-mm wirereinforced tracheal tubes, respectively (TUORen Medical Equipment Co., Henan, China. Figure 1, B) with high-volume, low-pressure cuffs. Anaesthesia was maintained with propofol, remifentanil and sevoflurane.

An aseptic suction catheter (OD - $4.0 \mathrm{~mm}$, Fig. 1, C) lubricated with aseptic paraffin was inserted through the tracheal tube with its tip protruding approximately $15 \mathrm{~cm}$ (Fig. 1, B), and the tube was then advanced through the nasopharynx. The Macintosh laryngoscope was then placed into the patient's mouth. Then, in Group $\mathrm{M}$, the tracheal tube was inserted into the trachea in a conventional technique. However, in Group T, a tube core bent to the physiological curve of the nasal cavity lubricated with aseptic liquid paraffin was inserted into the tracheal tube, and the tube core was then withdrawn after the tracheal tube was advanced through the glottis under direct vision. NTI of all patients was performed, if required, using Magill forceps. The entire intubation process is shown in Fig. 2 for Group T. All intubations were performed by an anaesthesiologist who was familiar with both techniques and had extensive experience. Minute adjustments to ventilation were performed to maintain end-tidal $\mathrm{CO}_{2}$ pressures at $35-45 \mathrm{mmHg}$ after intubation.

The NTI time, which was defined as the period from when the anaesthesiologist picked up the device to when three successive end-tidal $\mathrm{CO}_{2}$ waves were obtained following intubation[12], was recorded. 
Epistaxis was assessed by an observer blinded to the group assignments using direct laryngoscopy at five minutes after completing NTI and was scored as one of four grades according to the following modified criteria: no epistaxis (no blood observed on either the surface of the tube or the posterior pharyngeal wall); mild epistaxis (blood apparent on the surface of the tube or posterior pharyngeal wall); moderate epistaxis (pooling of blood on the posterior pharyngeal wall); and severe epistaxis (a large amount of blood in the pharynx that impeded NTI and necessitated urgent orotracheal intubation)[13]. The frequency of using Magill forceps in two groups was also recorded.

Neuromuscular blockade was reversed using neostigmine $1 \mathrm{mg}$ and atropine $0.5 \mathrm{mg}$, and the trachea was extubated when the patient was awake. An investigator who was blinded to the study assessed the nasal pain at $15 \mathrm{~min}, 1 \mathrm{~h}$, and $24 \mathrm{~h}$ after extubation on a visual analogue scale (VAS) according to a $10-\mathrm{cm}$ vertical score ranging from $0=$ no pain to $10=$ worst pain imaginable.

To calculate the sample size, we undertook a pilot study with 10 patients in each group (total 20 patients). The NTI time was significantly longer in the Magill forceps group than in the tube core group (Group M, 59.8 (5.1) sec vs Group T vs 53.1 (3.0) sec). For this study, the total sample size to achieve 0.95 power and an a-error of 0.05 was 12 patients per group according to $G *$ Power 3.1.9.4 software. Sixty adult patients who were rated as American Society of Anaesthesiologists (ASA) I or $\Pi$ and whose condition required NTI under general anaesthesia were selected.

Data are expressed as the mean (SD). Parametric data were compared between the groups by analysis of variance and post hoc testing. Categorical data were analysed using Fisher's exact test. Statistical significance was considered at $P$ values $<0.05$. All statistical analyses were performed with Statistical Package for Social Sciences (SPSS) software 20.0.

\section{Results}

Sixty patients were enrolled in our study. Figure 3 shows the CONSORT flow diagram for patient inclusion. There were no differences between the two groups with regard to demographic data (Table 1). 
Table 1

Patient characteristics and the duration of anaesthesia

\begin{tabular}{|llll|}
\hline Variable & Group M $(\boldsymbol{n}=\mathbf{3 0})$ & Group T $(\boldsymbol{n}=\mathbf{3 0})$ & $\boldsymbol{P}$ value \\
\hline Age (years) & $46.4 \pm 14.0$ & $49.9 \pm 17.7$ & 0.399 \\
\hline Height $(\mathrm{cm})$ & $166.3 \pm 6.7$ & $166.6 \pm 8.7$ & 0.855 \\
\hline Weight $(\mathrm{kg})$ & $67.2 \pm 11.2$ & $66.5 \pm 9.4$ & 0.794 \\
\hline ASA physical status $(\mathrm{I} / \Pi)$ & $12 / 18$ & $9 / 21$ & 0.417 \\
\hline Sex (male:female) & $17 / 13$ & $16 / 14$ & 0.795 \\
\hline BMI (kg $\cdot \mathrm{m}^{-2}$ ) & $24.2 \pm 3.1$ & $23.9 \pm 2.4$ & 0.679 \\
\hline The CL grading scale & $19 / 11$ & $17 / 13$ & 0.598 \\
\hline Duration of anaesthesia & $69.2 \pm 38.5$ & $85.5 \pm 42.1$ & 0.123 \\
(min) & & \\
\hline Values are expressed as a number or the mean (standard deviation) & \\
\hline
\end{tabular}

There was a significant difference in total NTI time between the two groups (Group M, 59.7 (5.1) sec vs Group T, 52.4 (3.1) sec) (Table 2).

Table 2

NTI time and associated complications

\begin{tabular}{|lccc|}
\hline Variable & $\begin{array}{l}\text { Group M } \\
(\boldsymbol{n = 3 0 )}\end{array}$ & $\begin{array}{l}\text { Group T } \\
(\boldsymbol{n}=\mathbf{3 0})\end{array}$ & Pvalue \\
\hline NTI time (sec) & $59.7 \pm 5.1$ & $52.4 \pm 3.1$ & 0.000 \\
Epistaxis( Mild/Moderate/Severe) & $6(6 / 0 / 0)$ & $5(5 / 0 / 0)$ & 0.739 \\
\hline \multicolumn{4}{|l|}{ Values are expressed as a number or the mean (standard deviation). } \\
\hline
\end{tabular}

Mild epistaxis was observed in 6 patients in Group M and 5 patients in Group T (6/30 vs 5/30, respectively). No moderate or severe epistaxis occurred in either group (Table 2). There was no presence of damaged oral tissue or damage to the teeth in either group.

All NTIs were completed successfully, and no Magill forceps were used in Group T but all were used in Group M. There was no obvious nasal pain at any time point after extubation in either group, which was similar to the result of our previous report; therefore, the data were not shown. Additionally, the degree of pain from sore throat in our study was not assessed because some of the surgeries were performed on the vocal cords. 


\section{Discussion}

Magill forceps are usually needed to facilitate insertion of the endotracheal tube into the glottis $[1-3,12$, $14,15]$. However, they may cause rupture of the cuff or mucosal injury and even lead to infection[5-7]. Moreover, this instrument is always not a disposable sterilised medical device and requires strict aseptic techniques after surgery. It was reported that the percentage of needing for Magill forceps with conventional technique closing to $70 \%[1]$, however, it was $100 \%$ in our study. This maybe because the Wire-reinforced tracheal tubes equipment used in our study were so soft that the tips of the tube couldn't be sent into glottis easily. In our present study, the tube core, a disposable sterilised stylet, could completely replace the use of Magill forceps in NTI and decrease the NTI time significantly. This process could significantly avoid infection caused by Magill forceps and make the process of NTI more convenient.

A systematic review has demonstrated that the risk of patient infection following a reusable device is significant, warranting a need for guidelines on reprocessing to be stricter to ensure greater patient safety, and indeed, when considering the risk of infection in the cost analysis, the findings from this study suggest benefits of disposable medical apparatuses in terms of cost effectiveness, cross-contamination and resource utilisation[16]. Miller et al.[6] found that many cleaning methods had not removed all proteinaceous material, which showed that even following the guidelines for cleaning the equipment may be insufficient to protect patients from transmission of iatrogenic disease, although methods and techniques have been further improved. Staining was even present in $60 \%$ of the Magill forceps group. Therefore, this situation prompted us to seriously question the safety of re-using instruments.

Perioperative infection has a significant impact on the outcome of surgical patients. Anaesthesiologists have roles in reducing infection by applying appropriate prophylactic measures[17]. The Magill forceps are not always disposable sterilised medical devices and require strict aseptic techniques after surgery. Perhaps we could consider the tube core as an optimal choice in NTI where possible.

It was reported that the wire tube body of the Disposcope endoscope was rigid but could be bent along the radian of the nasal cavity, which benefitted NTI[9]. Another device, a video intubating stylet for NTI, which has a rigid intubating stylet with an adjustable distal portion, makes the process of NTI quicker and easier[18]. In those studies, the tube stylet was advanced through into the glottis easily by their levelling effect but did not increase the incidence of related complications $[9,14,18]$. Although the tube core has a malleable rigid body, it protects the nasal mucosa and the entire nasal passage during the operational process by wire-reinforced tracheal tube, with results similar to those in our previous study[9].

Epistaxis, or postpharyngeal bleeding, is the most common complication after NTI. Thermosoftening of the endotracheal tube should not be overlooked even if other effective methods, such as telescoping the endotracheal tube into a rubber catheter, have already been applied because it has the obvious advantages of reducing the incidence of epistaxis and improving the nasal passage of the endotracheal tube $[3,15]$. The wire-reinforced tracheal tube in our study is a soft endotracheal tube that contributes to reducing bleeding[9]. Therefore, simple thermosoftening treatment of the endotracheal tube has not been 
performed in the present research. On the other hand, NTI under suction catheter guidance increases the success rate of airway instrumentation and reduces the incidence and severity of epistaxis[19]. All of these factors may have decreased the severity of epistaxis in our study.

To be sure, there are some limitations to our study. First, the operator was not blinded to the research, which may have affected the process of NTI. Second, the incidence of sore throat was not assessed because some of the surgeries were performed on the vocal cords. Third, patients with preoperative modified Mallampati scores of III or IV were excluded from our study, which should be addressed in our research in the future. Furthermore, the NTI was completed under a Macintosh laryngoscope in the present study. However, videolaryngoscopy is associated with a significantly decreased force exerted on maxillary incisors and might reduce the risk for dental injury in clinical settings[20]; moreover, it can clearly observe the glottis, which can simplify the process of NTI.

\section{Conclusion}

This study shows that using a tube core, a disposable sterilised stylet, could completely replace the use of Magill forceps in NTI and reduce the NTI time significantly without a difficult airway. Therefore, using a tube core for NTI without a difficult airway may be a convenient choice when possible.

\section{Abbreviations}

ASA

American Society of Anaesthesiologists

$\mathrm{BMI}$

body mass index

NTI

Nasotracheal intubation

VAS

visual analogue scale

\section{Declarations}

\section{Consort}

Our study adheres to CONSORT guidelines.

\section{Availability of data and materials}

The datasets analysed during the current study are available from the corresponding author upon reasonable request.

\section{Ethics approval and consent to participate}


This study protocol was approved by the Institutional Research Ethics Committee of The First People's Hospital of Hefei (No. 2019-12) and was registered in the Chinese Clinical Trial Registry (www.Chictr.org.cn, ChiCTR1900027387). Written informed consent was obtained from all patients, and the study was conducted in accordance with the Declaration of Helsinki.

\section{Consent for publication}

Not applicable.

\section{Availability of data and materials}

The datasets analysed during the current study are available from the corresponding author upon reasonable request.

\section{Competing interests}

The authors declare that they have no competing interests.

\section{Funding}

No funding was obtained for this study.

\section{Authors' contributions}

$\mathrm{RH}$ and JYN helped to design the study, conduct the study, collect the data, analyze the data, and write the manuscript. LNW, HS and PS helped to conduct the study and collect the data. JYH helped to conduct the study. JMY helped to design the study and write the manuscript. All authors have read and approved the manuscript submitted for publication.

\section{Acknowledgments}

The authors would like to thank all the staff of the Department of Anaesthesiology, The Third Affiliated Hospital of Anhui Medical University (The First People's Hospital of Hefei) and the Department of Otorhinolaryngology Surgery, The Third Affiliated Hospital of Anhui Medical University (The First People's Hospital of Hefei) for their help in finishing this research.

\section{References}

1. Lim CW, Min SW, Kim CS, Chang JE, Park JE, Hwang JY. The use of a nasogastric tube to facilitate nasotracheal intubation: a randomised controlled trial. Anaesthesia. 2014;69(6):591-7.

2. Pourfakhr P, Ahangari A, Etezadi F, Moharari RS, Ahmadi A, Saeedi N, Najafi A. Comparison of Nasal Intubations by GlideScope With and Without a Bougie Guide in Patients Who Underwent Maxillofacial Surgeries: Randomized Clinical Trial. Anesthesia analgesia. 2018;126(5):1641-5. 
3. Kim EM, Chung MH, Lee MH, Choi EM, Jun IJ, Yun TH, Ko YK, Kim JH, Jun JH. Is Tube Thermosoftening Helpful for Videolaryngoscope-Guided Nasotracheal Intubation?: A Randomized Controlled Trial. Anesthesia analgesia. 2019;129(3):812-8.

4. Abrons RO, Zimmerman MB, El-Hattab YMS. Nasotracheal intubation over a bougie vs. non-bougie intubation: a prospective randomised, controlled trial in older children and adults using videolaryngoscopy. Anaesthesia. 2017;72(12):1491-500.

5. Nakamura S, Watanabe T, Hiroi E, Sasaki T, Matsumoto N, Hori T. Cuff damage during naso-tracheal intubation for general anesthesia in oral surgery. Masui. 1997;46(11):1508-14.

6. Miller DM, Youkhana I, Karunaratne WU, Pearce A. Presence of protein deposits on 'cleaned' re-usable anaesthetic equipment. Anaesthesia. 2001;56(11):1069-72.

7. Mahajan R, Batra YK, Kumar S. Another use of Magill forceps to assist nasotracheal intubation. Can J Anaesth. 2007;54(11):957-8.

8. Kumar R, Gupta E, Kumar S, Rani Sharma K, Rani Gupta N. Cuff inflation-supplemented laryngoscope-guided nasal intubation: a comparison of three endotracheal tubes. Anesthesia analgesia. 2013;116(3):619-24.

9. Yu J, Hu R, Wu L, Sun P, Zhang Z. A comparison between the Disposcope endoscope and fibreoptic bronchoscope for nasotracheal intubation: a randomized controlled trial. BMC Anesthesiol. 2019;19(1):163.

10. Driver BE, Prekker ME, Klein LR, Reardon RF, Miner JR, Fagerstrom ET, Cleghorn MR, McGill JW, Cole JB. Effect of Use of a Bougie vs Endotracheal Tube and Stylet on First-Attempt Intubation Success Among Patients With Difficult Airways Undergoing Emergency Intubation: A Randomized Clinical Trial. JAMA. 2018;319(21):2179-89.

11. Cormack RS, Lehane J. Difficult tracheal intubation in obstetrics. Anaesthesia. 1984;39(11):110511.

12. Yeom JH, Oh MK, Shin WJ, Ahn DW, Jeon WJ, Cho SY. Randomized comparison of the effectiveness of nasal intubation using a GlideScope video laryngoscope with Magill forceps versus vascular forceps in patients with a normal airway. Can J Anaesth. 2017;64(12):1176-81.

13. Earle R, Shanahan E, Vaghadia H, Sawka A, Tang R. Epistaxis during nasotracheal intubation: a randomized trial of the Parker Flex-Tip nasal endotracheal tube with a posterior facing bevel versus a standard nasal RAE endotracheal tube. Can J Anaesth. 2017;64(4):370-5.

14. Staar S, Biesler I, Muller D, Pfortner R, Mohr C, Groeben H. Nasotracheal intubation with three indirect laryngoscopes assisted by standard or modified Magill forceps. Anaesthesia. 2013;68(5):467-71.

15. Kim YC, Lee SH, Noh GJ, Cho SY, Yeom JH, Shin WJ, Lee DH, Ryu JS, Park YS, Cha KJ, et al. Thermosoftening treatment of the nasotracheal tube before intubation can reduce epistaxis and nasal damage. Anesthesia analgesia. 2000;91(3):698-701.

16. Mouritsen JM, Ehlers L, Kovaleva J, Ahmad I, El-Boghdadly K. A systematic review and cost effectiveness analysis of reusable vs. single-use flexible bronchoscopes. Anaesthesia. 2020;75(4):529-40. 
17. Shime N. Role of anesthesiologist in prevention of perioperative infection. Masui. 2014;63(3):26977.

18. Lee MC, Tseng KY, Shen YC, Lin CH, Hsu CW, Hsu HJ, Lu IC, Cheng KI. Nasotracheal intubation in patients with limited mouth opening: a comparison between fibreoptic intubation and the Trachway(R). Anaesthesia. 2016;71(1):31-8.

19. Morimoto Y, Sugimura M, Hirose Y, Taki K, Niwa H. Nasotracheal intubation under curve-tipped suction catheter guidance reduces epistaxis. Can J Anaesth. 2006;53(3):295-8.

20. Schieren M, Kleinschmidt J, Schmutz A, Loop T, Staat M, Gatzweiler KH, Wappler F, Defosse J. Comparison of forces acting on maxillary incisors during tracheal intubation with different laryngoscopy techniques: a blinded manikin study. Anaesthesia. 2019;74(12):1563-71.

\section{Figures}
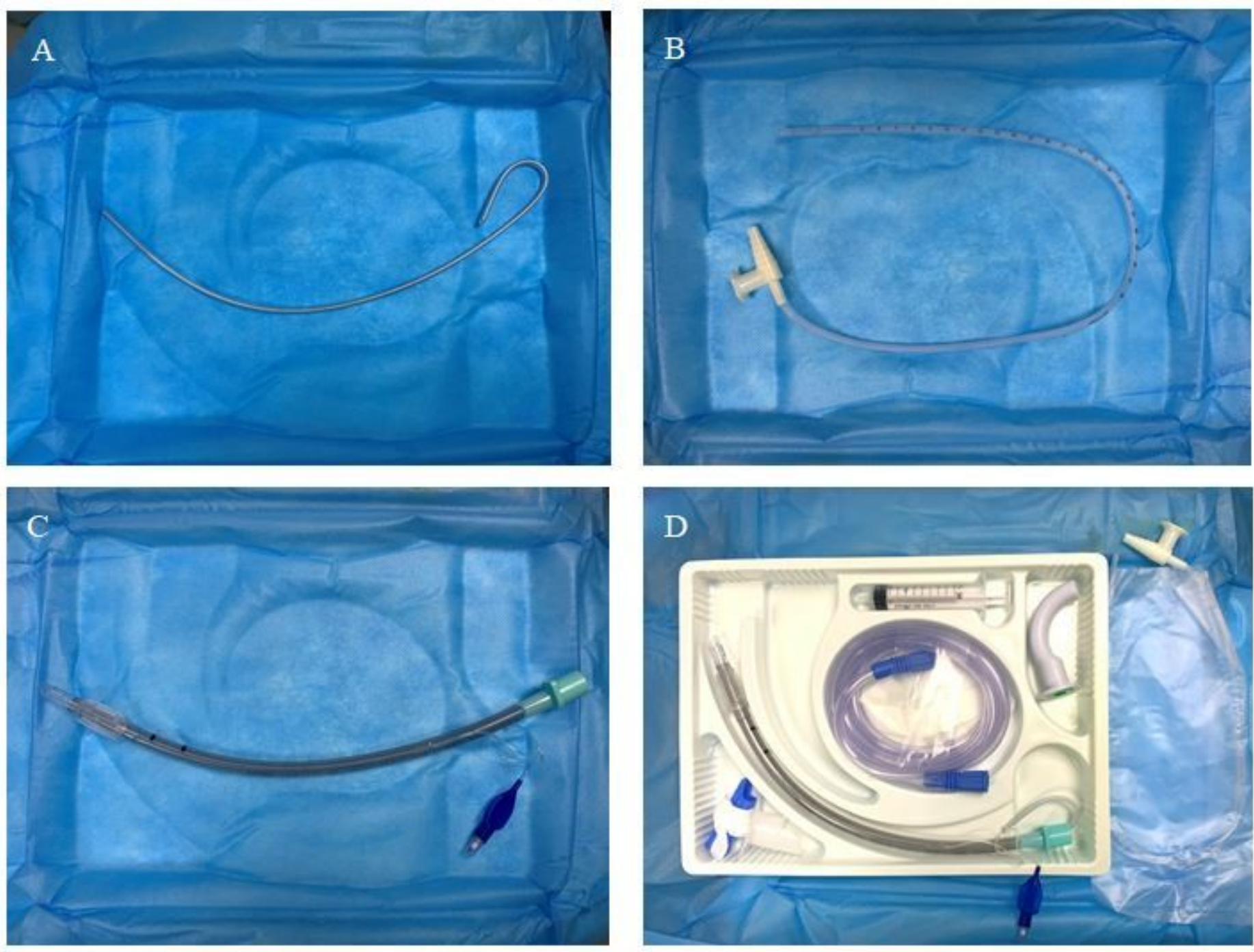

\section{Figure 1}


Tube core, a malleable rigid stylet (A); Wire-reinforced tracheal tube (B); Aseptic suction catheter (OD - 4.0 $\mathrm{mm}, \mathrm{C}$ ); Wire-reinforced tracheal tubes equipment (TUORen Medical Equipment Co., Henan, China. D).

A

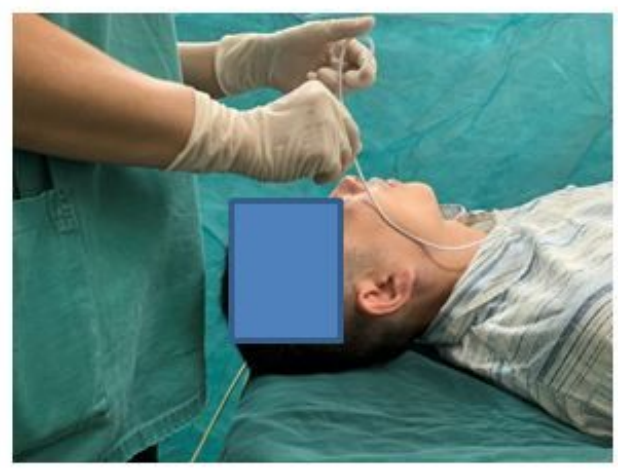

\section{$\mathrm{D}$}

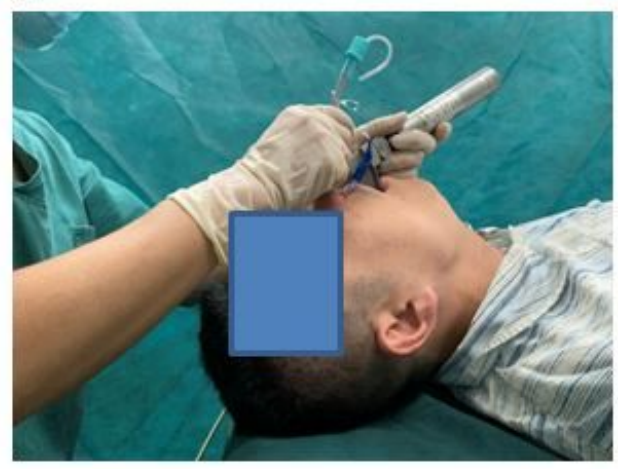

$\mathrm{B}$

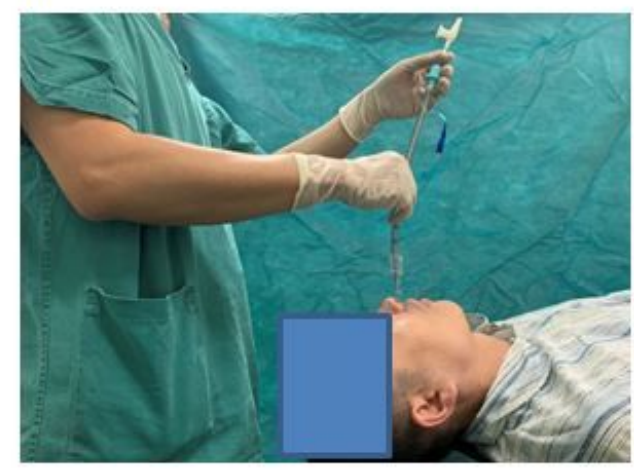

E

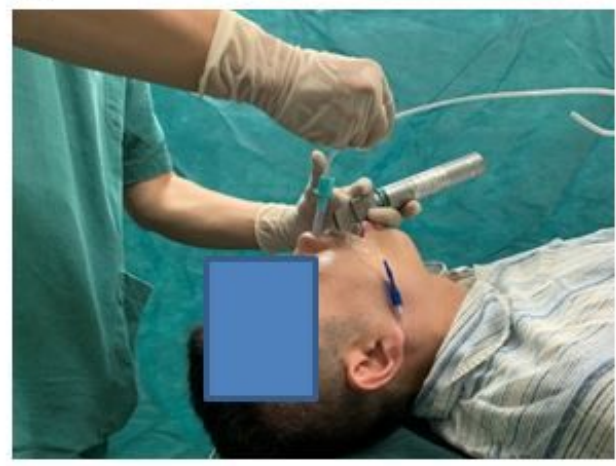

$\mathrm{C}$

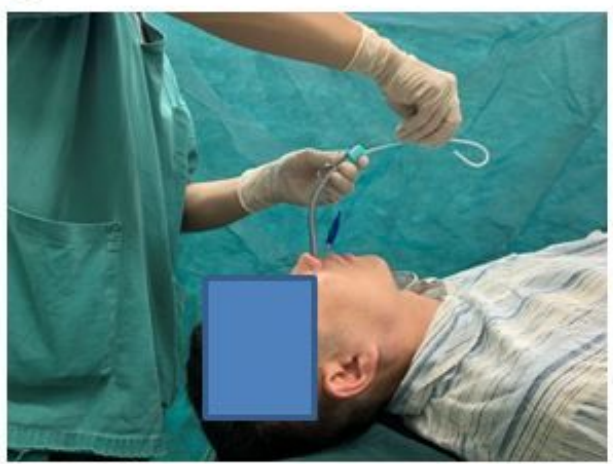

F

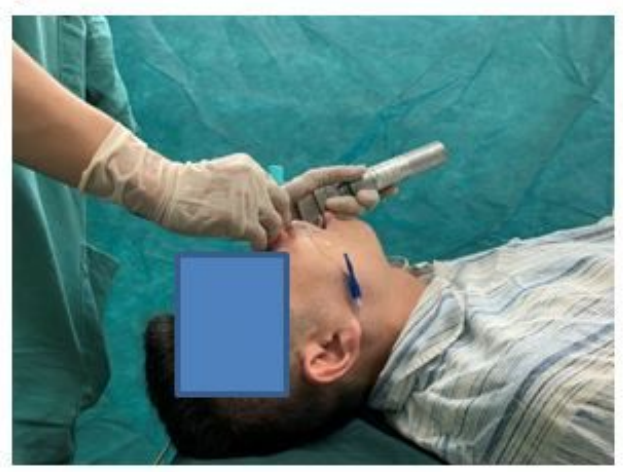

\section{Figure 2}

The entire process for nasotracheal intubation (A-F). (A) The tube core was bent along the radian of the nasal cavity. (B) The tracheal tube was inserted through the nasopharynx under aseptic suction catheter guidance. ( $\mathrm{C}$ to $\mathrm{F}$ ) Nasotracheal intubation was performed using a tube core after the suction catheter was withdrawn. 


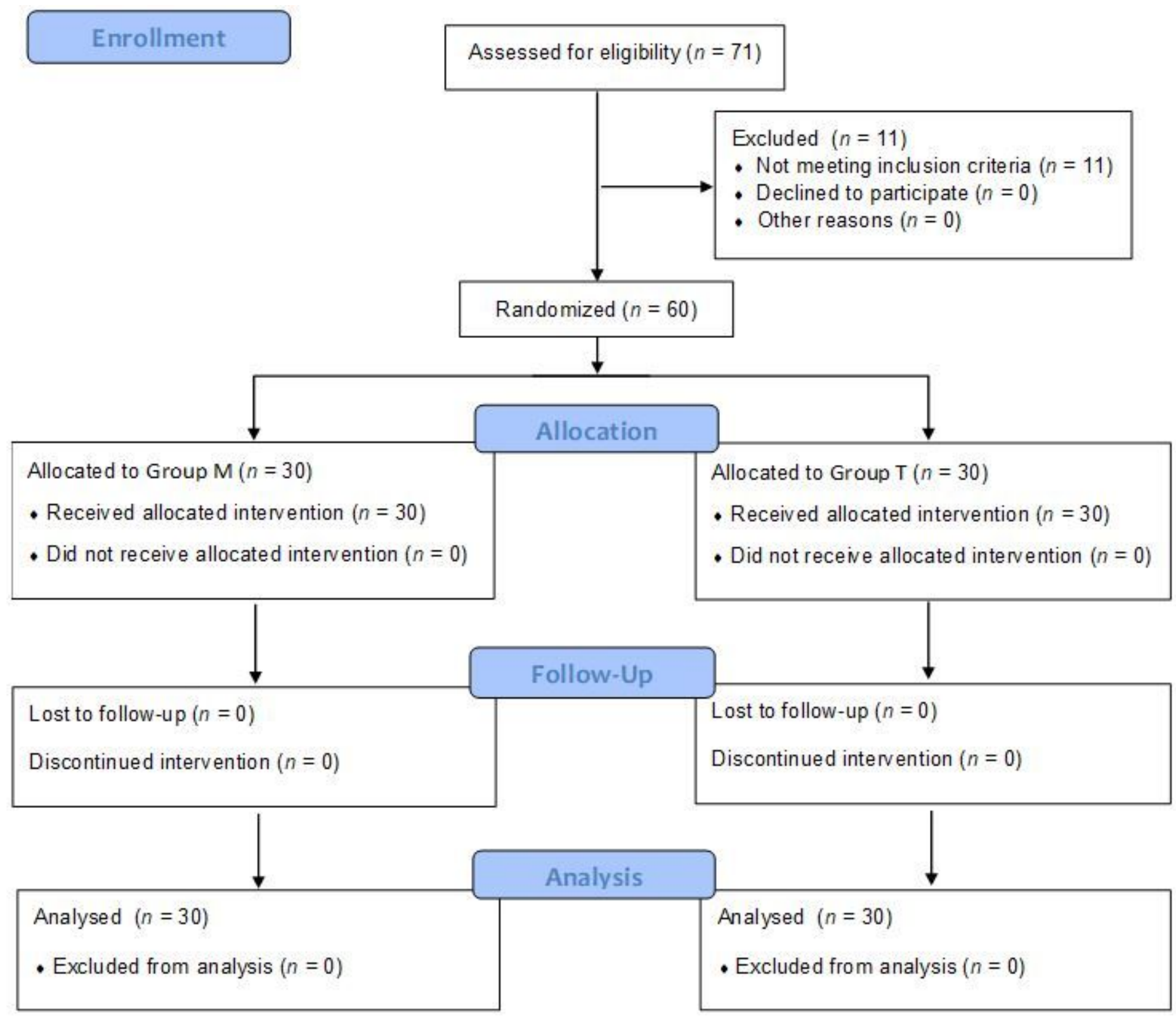

\section{Figure 3}

Flow chart illustrating the recruitment and loss of patients in Group M and Group T.

\section{Supplementary Files}

This is a list of supplementary files associated with this preprint. Click to download.

- CONSORT2010Checklist.doc 